\title{
BMJ Open Study protocol of a randomised controlled trial of prostate radiotherapy in high-risk and node-positive disease comparing moderate and extreme hypofractionation (PRIME TRIAL)
}

To cite: Murthy V, Mallick I, Gavarraju A, et al. Study protocol of a randomised controlled trial of prostate radiotherapy in high-risk and node-positive disease comparing moderate and extreme hypofractionation (PRIME TRIAL). BMJ Open 2020;10:e034623. doi:10.1136/ bmjopen-2019-034623

- Prepublication history and additional material for this paper are available online. To view these files, please visit the journal online (http://dx.doi. org/10.1136/bmjopen-2019034623).

Received 02 0ctober 2019 Revised 15 January 2020 Accepted 23 January 2020

Check for updates

(C) Author(s) (or their employer(s)) 2020. Re-use permitted under CC BY-NC. No commercial re-use. See rights and permissions. Published by BMJ.

For numbered affiliations see end of article.

Correspondence to Dr Vedang Murthy; vmurthy@actrec.gov.in

\section{ABSTRACT}

Introduction There has been an interest in studying the efficacy of extreme hypofractionation in low and intermediate risk prostate cancer utilising the low alpha/ beta ratio of prostate. Its role in high-risk and nodepositive prostate cancer, however, is unknown. We hypothesise that a five-fraction schedule of extreme hypofractionation will be non-inferior to a moderately hypofractionated regimen over 5 weeks in efficacy and will have acceptable toxicity and quality of life while reducing the cost implications during treatment.

Methods and analysis This is an ongoing, non-inferiority, multicentre, randomised trial (NCT03561961) of two schedules for National Cancer Control Network high-risk and/or node-positive non-metastatic carcinoma of the prostate. The standard arm will be a schedule of $68 \mathrm{~Gy} / 25 \#$ over 5 weeks while the test arm will be extremely hypofractionated radiotherapy with stereotactic body radiation therapy to $36.25 \mathrm{~Gy} / 5 \#$ (7 to 10 days). The block randomisation will be stratified by nodal status $(\mathrm{NO} / \mathrm{N}+)$, hormonal therapy (luteinizing hormone-releasing hormone therapy/orchiectomy) and centre. All patients will receive daily image-guided radiotherapy.

The primary end point is 4-year biochemical failure free survival (BFFS). The power calculations assume 4-year BFFS of $80 \%$ in the moderate hypofractionation arm. With a $5 \%$ one-sided significance and $80 \%$ power, a total of 434 patients will be randomised to both arms equally (217 in each arm). The secondary end points include overall survival, prostate cancer specific survival, acute and late toxicities, quality of life and out-of-pocket expenditure.

Discussion The trial aims to establish a therapeutically efficacious and cost-efficient modality for high-risk and node-positive prostate cancer with an acceptable toxicity profile. Presently, this is the only trial evaluating and answering such a question in this cohort.

Ethics and dissemination The trial has been approved by IEC-III of Tata Memorial Centre, Mumbai.

Trial registration number Registered with CTRI/2018/05/014054 (http://ctri.nic.in) on 24 May 2018

\section{Strengths and limitations of this study}

- First study addressing the role of moderate hypofractionation and stereotactic body radiation therapy (SBRT) in high-risk and node-positive prostate cancer.

- Use of prostate-specific membrane antigen positron emission tomography/CT for all patients at baseline for staging and risk adapted approach.

- Out-of-pocket expenditure will be collected from all patients which will potentially have a huge impact on logistics and resource utilisation especially in low-middle-income country like India.

- Non-inferiority margin between standard arm and test arm kept at $9 \%$.

- No published evidence for role of SBRT in nodepositive prostate cancer in the literature.

\section{INTRODUCTION}

Incidence of high-risk/very high-risk prostate cancer is on a rising trend across the globe. ${ }^{1-4}$ Advanced prostate cancer accounts for $15 \%$ of all diagnosed prostate cancers in developed nations whereas in low and low-middleincome countries like India the proportion of advanced disease is estimated to be as high as $84 \% .{ }^{5}$ Radiotherapy in the form of external beam radiotherapy (EBRT) and/ or brachytherapy forms an integral part of management of these patients. Radiobiological studies have shown that prostate cancer has a low alpha/beta ratio in the range of 0.47 to $4.14 .^{6}$ This makes hypofractionated radiotherapy radiobiologically superior than conventional fractionated (treatment time 7 to 8 weeks) schedules as it leads to a considerably higher biologically equivalent dose delivery. Prospective randomised trials have 
studied the safety and efficacy of moderate hypofractionation (treatment time 4 to 6 weeks) in prostate cancer and is now considered as the standard of care in low-risk and intermediate-risk prostate cancer. ${ }^{7}$ With the benefit shown with moderate hypofractionation, there has been a growing interest in the role of extreme hypofractionation in prostate cancer.

Extreme hypofractionation (treatment duration 7 to 10 days) with stereotactic body radiation therapy (SBRT) has an emerging role as an alternative technique to deliver high-dose radiotherapy to the prostate comparable to brachytherapy, but with a non-invasive approach. However, the acute and late toxicities remain a concern with SBRT. Multiple single arm series on the use of SBRT as the primary treatment for prostate cancer have suggested the treatment to be safe but these studies have a majority of patients from low/intermediate risk group. The results of HYPO-RT-PC trial published recently supports the safety of SBRT in low-risk/intermediate-risk prostate cancer. ${ }^{8}$

The data with regards to extreme hypofractionation for high-risk prostate cancer is still sparse. Recently, the early toxicity and quality of life results of a phase I/II study of stereotactic ablative radiotherapy including regional lymph node irradiation in patients with high-risk prostate cancer (SATURN) has demonstrated the safety of SBRT for these patients. ${ }^{9}$ Similarly, a retrospective series of 68 patients reported from India reported equivalent toxicities as compared with moderate hypofractionation in this subset of patients. ${ }^{10}$

Extreme hypofractionation (SBRT) for a total duration of 7 to 10 days, would offer an opportunity to optimise the therapeutic ratio for the treatment of these tumours along with significantly decreasing the overall treatment time which in turn would lead to significantly better quality of life during treatment, early recommencement to daily activities while reducing the financial burden for these patients. We therefore initiated a randomised phase III trial to establish the non-inferiority of SBRT in high-risk and/or node-positive prostate cancer (NCT03561961).

\section{METHODS/DESIGN \\ Trial design}

This is an ongoing, prospective, multicentre, two arm randomised control trial with a non-inferiority design led from a tertiary care cancer centre in India for highrisk node-negative and node-positive prostate cancer patients. Randomisation will be stratified by randomisation method in a 1:1 ratio.

1. Standard arm/moderate hypofractionation: Treatment duration, 5 weeks.

2. Test arm/extreme hypofractionation: Treatment duration, 7 to 10 days.

Stratification will be done for the following parameters:

1. Nodal status: $\mathrm{N} 0$ vs $\mathrm{N}+$.

2. luteinizing hormone-releasing hormone (LHRH) agonist/antagonists versus bilateral orchiectomy.
3. Centre.

The inclusion criteria for the trial will include patients who are older than 18 years with histologically proven adenocarcinoma prostate localised to prostate and pelvic nodes with high-risk/very high-risk disease as per National Cancer Control Network risk stratification (clinical stage T3a or Gleason score 8/Gleason grade group 4 or Gleason score 9 to 10/Gleason grade group 5, prostate-specific antigen (PSA) $>20 \mathrm{ng} / \mathrm{mL}$ or very highrisk prostate cancer, that is, T3b/T4 or primary Gleason pattern 5/Gleason grade group 5 or $>4$ cores, Gleason score 8 to $10 /$ Gleason grade group 4 or 5 ). All patients will be staged with a baseline prostate-specific membrane antigen (PSMA) positron emission tomography (PET)/ CT. In addition, the patients should be fit to receive longterm androgen deprivation therapy in the form of either orchiectomy/hormonal therapy (2 years) and have a baseline Karnofsky Performance Scale of more than equal to 70 (online supplementary appendix 1). The exclusion criteria for the trial includes a distant metastatic disease, a life expectancy of less than 2 years, patient's with previous history of pelvic radiotherapy (RT), patients with severe urinary symptoms (International Prostate Symptom Score (IPSS) >15) despite being on hormone therapy for 6 months, patients with obstructive urinary symptoms like stricture, patients with contraindication to EBRT like pelvic inflammatory disease and patients with uncontrolled comorbidities (table 1 ).

\section{Patient and public involvement}

Patient/public were not involved in the research methodology of this ongoing study. All patients were given an informed consent form. Only after willingly consenting and understanding all the aspects of participation in the trial, patients were randomised to either of the two arms.

\section{Baseline evaluation and radiotherapy details}

The baseline evaluation would include standard workup for a locally advanced carcinoma prostate, that is, transrectal ultrasonography-guided 12 core biopsy and baseline serum PSA level. PSMA PET/CT will be used at baseline to stage all patients. Nodal involvement by disease will be defined as maximum standardised uptake value $\geq 3.0$ irrespective of the size but based on morphological characteristics like shape, heterogeneity and perinodal stranding as determined by expert in uroradiology (PP) and nuclear imaging (VR, AA).

The duration of neoadjuvant androgen deprivation therapy (ADT) ranges from 8 to 12 weeks for nodenegative patients and 6 months for node-positive patients. Preferably, all patients will have baseline documentation of quality of life (using European Organisation for Research and Treatment of Cancer Quality of Life Questionnaire C30 and PR25 questionnaire) and IPSS score at baseline as well as before starting EBRT.

In the standard arm, patients who are randomised to receive moderately hypofractionated RT will receive a total dose of $68 \mathrm{~Gy}$ in 25 fractions to the primary 
Table 1 Inclusion and exclusion criteria

\begin{tabular}{|c|c|}
\hline Inclusion criteria & Exclusion criteria \\
\hline 1. Age: above 18 years & 1. Evidence of distant metastasis at any time since presentation \\
\hline \multirow{2}{*}{$\begin{array}{l}\text { 2. Participants must be histologically proven, } \\
\text { adenocarcinoma prostate }\end{array}$} & 2. Life expectancy $<2$ years \\
\hline & 3. Previous RT to prostate or prostatectomy \\
\hline 3. Localised to the prostate or pelvic lymph nodes & 4. Severe urinary symptoms or with severe IPSS score $(>15)$ \\
\hline 4. High-risk prostate cancer as per NCCN definition & despite being on hormonal therapy for 6 months which in the \\
\hline 5. PSMA/PET CT for all patients at baseline for staging & opinion of the physician precludes RT \\
\hline 8. No prior history of therapeutic irradiation to pelvis & 7. Uncontrolled comorbidities including, but not limited to \\
\hline 9. Patient willing and reliable for follow-up & diabetes or hypertension \\
\hline 10. Signed study specific consent form & 8. Unable to follow-up or poor logistic or social support \\
\hline
\end{tabular}

IPSS, International Prostate Symptom Score; KPS, Karnofsky Performance Scale; NCCN, National Cancer Control Network; PET, positron emission tomography; PSMA, prostate-specific membrane antigen; RT, radiotherapy.

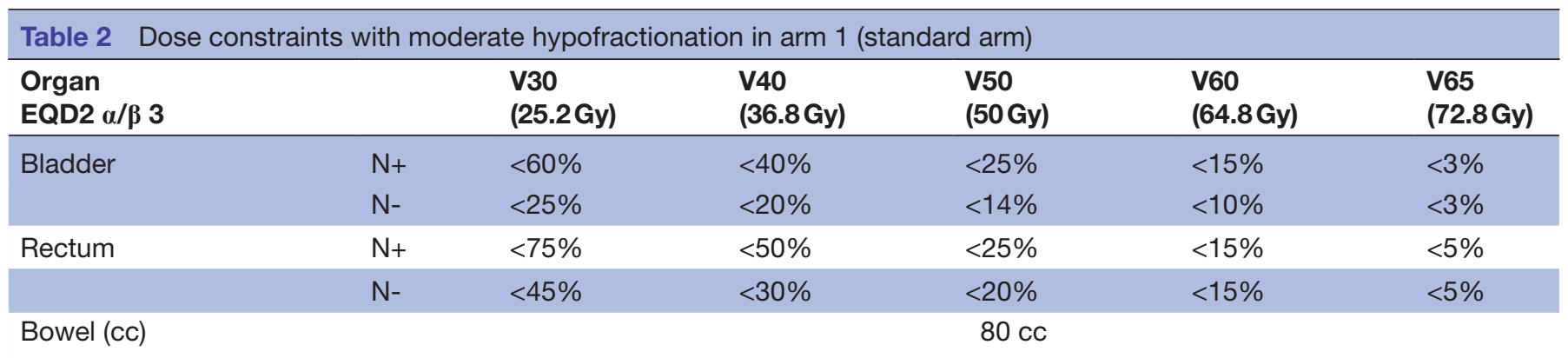

EQD2, Equivalent dose at 2 Gy per fraction.

over 5 weeks, with treatment being delivered daily. Patients with node-positive disease will receive a dose of $50 \mathrm{~Gy}$ in 25 fractions to the pelvis. Response assessment PSMA PET/CT will be done for all patients with pelvic nodal disease to ascertain the response to ADT based on morphology of the residual node and metabolic uptake as defined by the uroradiologist/nuclear medicine specialist. Patients with persistent residual nodal disease will be considered for nodal boost. Boost to gross nodal disease will be considered based on the response to hormonal therapy to a dose of 60 to $66 \mathrm{~Gy}$ in 25 fractions as a simultaneous integrated boost. An option of equivalent biological dose using 60 to
$62.5 \mathrm{~Gy}$ in 20 fractions may be allowed for multicentric accrual in the future. In the test arm of study, patients who are scheduled to receive extreme hypofractionated RT (SBRT) will receive a course of five fractions of radiation; each fraction size will be $7.25 \mathrm{~Gy}$. The total dose will be 36.25 Gy. Patients with node-positive disease will receive a dose of $25 \mathrm{~Gy}$ in five fractions to the pelvis. Boost to gross nodal disease will be considered based on the response to hormonal therapy to a dose of 30 to $35 \mathrm{~Gy}$ in five fractions as a simultaneous integrated boost. The five treatments will be scheduled to be delivered alternate day over approximately 7 to 10 days. An option of equivalent biological dose using

\begin{tabular}{|c|c|c|c|c|c|}
\hline & $\begin{array}{l}\text { V14 } \\
\text { (16.2Gy) }\end{array}$ & $\begin{array}{l}\text { V17.5 } \\
\text { (22.8Gy) }\end{array}$ & $\begin{array}{l}\text { V28 } \\
\text { (48.2 Gy) }\end{array}$ & $\begin{array}{l}\text { V31.5 } \\
\text { (58.6 Gy) }\end{array}$ & $\begin{array}{l}\text { V35 } \\
\text { (70 Gy) }\end{array}$ \\
\hline $\mathrm{N}-$ & $<35 \%$ & $<20 \%$ & $<10 \%$ & - & $<3 \%$ \\
\hline $\mathrm{N}+$ & $<50 \%$ & $<40 \%$ & $<15 \%$ & $<8 \%$ & $<3 \%$ \\
\hline & & & $80 \mathrm{cc}$ & & \\
\hline
\end{tabular}

EQD2, Equivalent dose at 2Gy per fraction. 


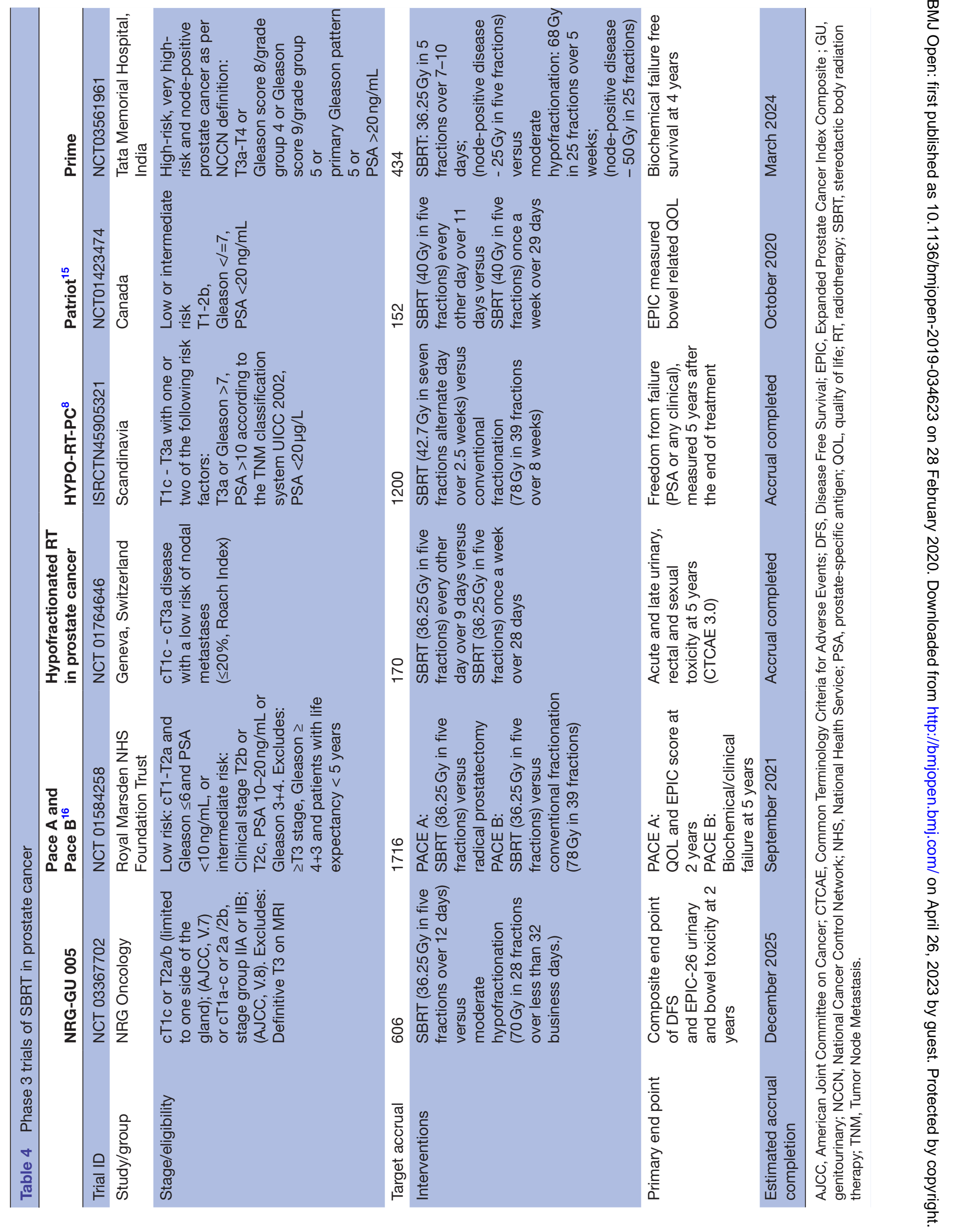




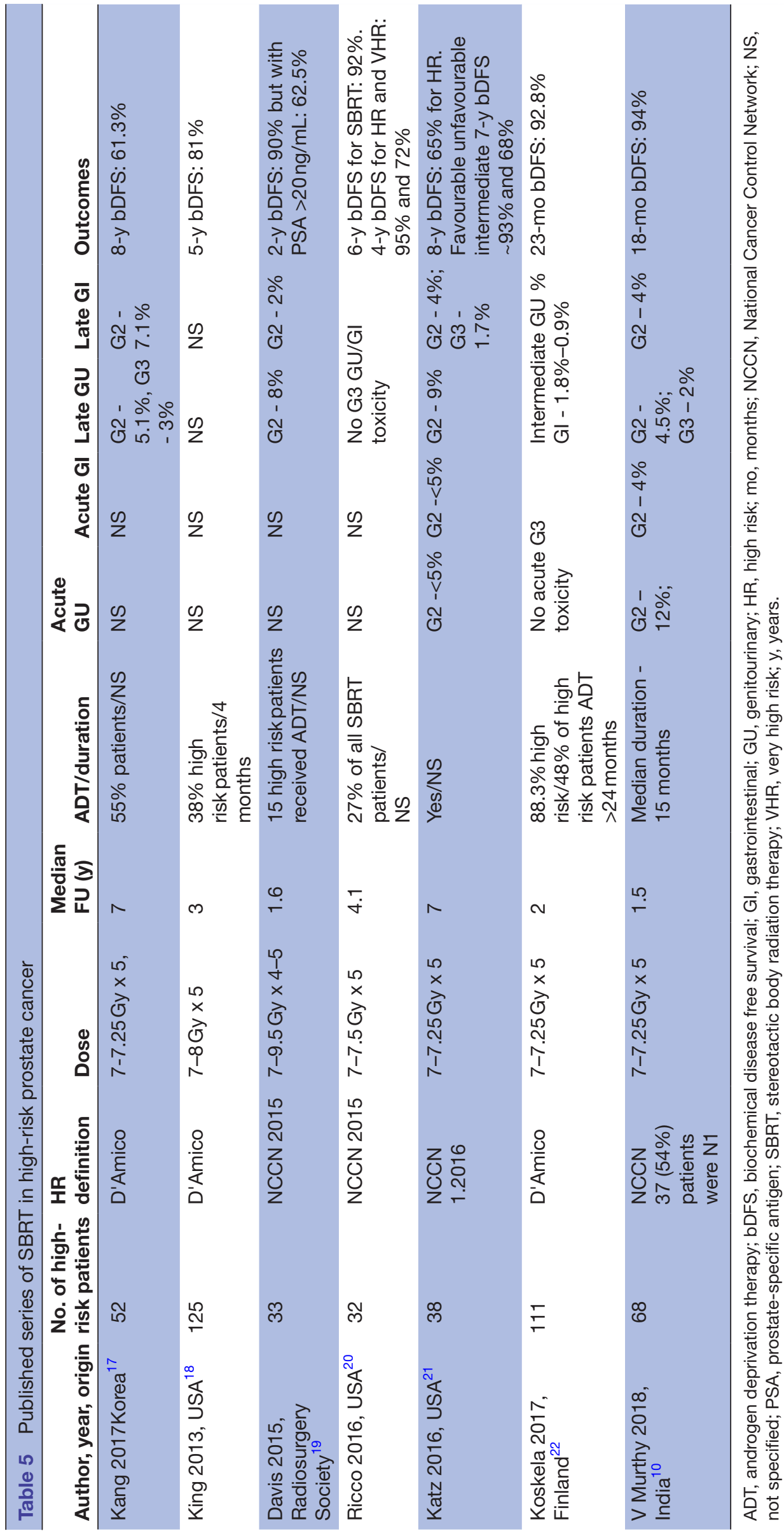


35 to 36.25 Gy in five weekly fractions may be allowed as per institutional practice for multicentric accrual in the future.

All patients will be treated with intensity-modulated radiation therapy or related techniques (helical tomotherapy/volumetric modulated arc therapy) with daily image guidance in the form of kilovoltage-cone beam CT (KV-CBCT)/megavoltage CT. No intraprostatic fiducials will be used for image-guided radiotherapy (IGRT). The contouring of gross tumour volume nodes, clinical target volume (CTV) primary and organs at risk will be done according to standard ESTRO ACROP guidelines. ${ }^{11}$ CTV nodes will be contoured by giving a radial margin of 5 to $7 \mathrm{~mm}$ around the common iliac, external iliac, internal iliac, presacral and the obturator vessels and editing from muscles and bones. The cranial extent of CTV nodes will be at the level of L5-S1 vertebra and the caudal extent will be at the level obturator nodes. For patients without seminal vesicle involvement only $1.5 \mathrm{~cm}$ of the base of seminal vesicles will be included in CTV primary whereas the entire seminal vesicle will be included in the CTV primary in patients with radiological involvement of seminal vesicle. A planning target volume (PTV) margins of $5 \mathrm{~mm}$ will be given around the entire CTV primary (including seminal vesicles) and CTV nodes to delineate PTV primary and PTV nodes, respectively. The dose volume constraints for the target volume will include a CTV D98 of 98\% (both primary and nodes) and a PTV D98 of 95\% (both primary and nodes). These contouring guidelines and dose constraints are in accordance with the retrospective series of SBRT for high-risk and/or node-positive prostate cancer published from the same institute. ${ }^{1012}$

The dose volume constraints for organs at risk is given in tables 2 and 3.

\section{Assessment/follow-up}

Patients will continue (LHRH agonist/antagonist) during radiotherapy. All patients will be monitored during radiotherapy and at every follow-up for acute/late toxicities using Radiation Therapy Oncology Group (RTOG) and Common Terminology Criteria for Adverse Events (CTCAE) 4.03 toxicity grading scales (online supplementary appendix 2-4). In addition, out-of-pocket expenditure (online supplementary appendix 5) of the patient and caregiver for food, travel, stay and for management of treatment related side effects will be captured using a structured record form along with quality of life score, IPSS score and urinary function. All patients will be followed up after 4 to 6 weeks of completion of radiotherapy and then at 3 to 6 monthly intervals until 2 years and 6 monthly intervals thereafter. Patients will continue LHRH agonists/antagonist after completion of radiotherapy to complete a total duration of 2 years. Clinical evaluation of the disease will be done at each follow-up visit with a serum PSA and clinical examination. Any other investigation will be done at the physician's discretion.

\section{Statistics}

The primary end point of this study will be 4-year biochemical failure free survival (BFFS) which will be defined as the time (in months) from the date of randomisation to the date of biochemical failure as per Phoenix Criteria (a rise in PSA level of more than $2 \mathrm{ng} / \mathrm{mL}$ above the nadir). This is a non-inferiority trial and the non-inferiority margin between the standard and the test arms is $9 \%$ (delta). To detect this delta difference in the primary end point of a 4-year BFFS (80\% in standard arm and $71 \%$ in the test arm; HR 1.53), with a power of $80 \%$ and a onesided 5\% alpha value, total of 135 events are required, with a minimum number of 422 patients. The assumption of $80 \%$ BFFS in the standard arm was based on the results of similar studies of high-risk node-negative cancer studies (STAMPEDE, James ND et al JAMA oncology 2016 and PRO 7, J Clin Oncol 2015) and our own data (unpublished) which showed a 5 year BFFS of about $85 \%$. As this study is recruiting high-risk and node-positive patients also, the upper limit of $80 \%$ was chosen. Non-inferiority of extreme hypofractionation with SBRT (test arm) versus moderate hypofractionated RT (standard arm) will be concluded if the upper limit of the estimated $95 \%$ onesided CI of the HR obtained lies entirely below 1.53. If the upper limit of the $95 \%$ one-sided CI is 1.53 or higher, we do not reject the null hypothesis of inferiority. The trial will accrue patients over a period of 6 years and all patients will be followed until the end of study. Considering an attrition rate of around 5\%, the trial would require a total of 434 patients (217 in each arm). The primary efficacy analysis will be on both intention-to-treat basis and per-protocol basis.

The secondary end points for the study will be evaluation of the acute and late toxicities (according to RTOG and CTCAE criterion), ascertaining the overall survival and prostate cancer specific survival for these patients, estimation of out-of-pocket expenditure involved in patients receiving the two treatment schedules and assessment of pre-treatment and post-treatment quality of life of these patients. For this study, overall survival and prostate cancer specific survival will be defined as time in months between date of randomisation and date of death due to any cause or date of death due to prostate cancer, respectively.

A planned interim analyses will be done on accrual of $25 \%$ (108 patients in both arms combined) patients completing 2 years of follow-up. At the interim analysis, the $\mathrm{p}$ value from the $\mathrm{X}^{2}$ or Fisher's exact test assessing treatment efficacy with respect to grade III or higher combined gastrointestinal (GI) and genitourinary (GU) RTOG toxicity will be compared in the two arms at one-sided alpha of $2.5 \%$ and a power of $80 \%$. If the computed $\mathrm{p}$ value is less than or equal to 0.025 , then accrual to the trial will be discussed with the Data Safety and Monitoring Committee for stopping (if applicable). Otherwise, accrual to the trial or follow-up (as applicable) will continue until the planned sample size $(n=434)$. We expect 80 patients to be accrued per year in the project 
with total study duration of about 6 years, with a non-fixed follow-up period and a uniform accrual rate.

\section{Quality assurance}

Radiation therapy quality assurance in the setting of a multi-institutional clinical trial has been shown to have a very important bearing on outcomes. The US National Cancer Institute Work Group on Radiotherapy Quality Assurance has laid down guidelines that help individual trials lay down their quality assurance (QA) protocol (Bekelman et al 2012). ${ }^{13}$ The PRIME trial will use these guidelines to formulate a trial specific protocol as outlined below.

\section{Tiered system for radiotherapy trial QA}

Tier 1 includes general credentialing which comprises of filling a facility questionnaire outlining machine-specific and patient-specific QA process.

Tier 2 is a trial specific credentialing consists of a dry run process with oncologists and physicists familiarising themselves with the contouring protocol and advanced dosimetry checks with multiple plan generation to meet trial constraints.

Tier 3 is an individualised case review of dosimetry and QA data sheet reviewed every monthly in virtual QA meet.

\section{DISCUSSION}

The efficacy of SBRT for prostate cancer is supported by a radiobiological basis as well as phase 1 and phase 2 single arm studies. Several single arm series have established the efficacy and safety of SBRT for low-risk and intermediate-risk prostate cancer. The results of ongoing phase 3 trials comparing extreme hypofractionation to moderate hypofractionation/conventional fractionation are awaited. (table 4). The major concern with SBRT is the impact on acute and late toxicities of the patients. The HYPO-RT-PC trial accrued 1200 intermediate-risk prostate cancer patients (T1c to T3a, PSA $</=20$, with one or two of the following risk factors: T3a or Gleason 7 or PSA $>10$ ) and tested conventionally fractionated RT against extreme hypofractionation. Preliminary results show no significant differences in the prevalence of physician reported grade $2+$ toxicity at 2 years between the two arms for urinary $(5.4 \%$ vs $4.6 \%, \mathrm{p}=0.59)$ and bowel $(2.2 \%$ vs $3.7 \%, p=0.20$ ) toxicity. ${ }^{8}$

The reports for SBRT as monotherapy for high-risk disease are limited as most contemporary series have included very few high-risk prostate cancers (table 5). The dose per fraction given in these series range from 7 to $8 \mathrm{~Gy}$ in four to fix fractions given over 7 to 14 days. The toxicities and outcomes are comparable to that expected with moderate hypofraction schedules with the GI and GU RTOG $\geq 2$ late toxicities ranging from $2 \%$ to $14 \%$.

FASTR is a phase 1 feasibility study for SBRT for nodepositive disease which delivered a dose of $25 \mathrm{~Gy}$ to pelvic nodes and $40 \mathrm{~Gy}$ to primary over a period of 5 weeks (once weekly fractionation). Nine out of the 15 patients accrued
$(60 \%)$ developed $\geq \mathrm{Gr}$ (grade) $2 \mathrm{GI}$ or GU toxicity at 6 months and $4(30 \%) \geq \mathrm{Gr} 3 \mathrm{GI}$ or GU toxicity at 6 months. The study was terminated before phase 2 in view of the higher toxicities. ${ }^{14}$ Another single arm study (SATURN) evaluated a similar protocol with a dose of $25 \mathrm{~Gy}$ to pelvic nodes and seminal vesicle, $40 \mathrm{~Gy}$ as simultaneous integrated boost to the prostate and 33.25 Gy to the prostate PTV delivered over a period of 5 weeks in five once weekly fractions. The authors reported a Gr $2 \mathrm{GU}$ toxicity of $52 \%$ (baseline 30\%) and 32\% GI toxicity (baseline 3.3\%) at 6 months. No grade 3 toxicities were reported. The authors attributed the lesser toxicity of this study as compared with the FASTR protocol to the lesser dose prescription to the prostate PTV ( 40 vs 33.25 Gy), the lesser PTV margins ( $5 \mathrm{~mm}$ vs $3 \mathrm{~mm}$ ), smaller CTV volumes and better image guidance in SATURN protocol. $^{9}$

In the present trial, the dose to primary PTV will be $36.25 \mathrm{~Gy}$ in five fractions, which is between those given in FASTR and SATURN trials. The efficacy and safety of such a dosing schedule has already been published as a single arm retrospective series from India with an 18-month biochemical disease free survival of $94 \%$ and incidence of $\geq \mathrm{Gr} 3$ late GU and GI toxicity of $3 \%$ and $0 \%$, respectively. In addition, the PTV margins are $5 \mathrm{~mm}$ which again has been shown to adequate with daily CBCT based IGRT. ${ }^{15}$

This trial aims to establish a therapeutically efficacious and cost-efficient modality for high-risk, very high-risk and node-positive prostate cancer which is likely to be a big public health problem in low-income/low-middleincome countries in the coming decade. Presently, this is the only trial evaluating and answering such a question in this cohort.

\begin{abstract}
Author affiliations
${ }^{1}$ Department of Radiation Oncology, Tata Memorial Centre, Mumbai, India

${ }^{2}$ Department of Radiation Oncology, Tata Medical Centre, Kolkata, India

${ }^{3}$ Clinical Research Secretariat, Tata Memorial Centre, Mumbai, India

${ }^{4}$ Division of Uro-Oncology, Tata Memorial Centre, Mumbai, India

${ }^{5}$ Department of Pathology, Tata Memorial Centre, Mumbai, India

${ }^{6}$ Department of Radiology, Tata Memorial Centre, Mumbai, India

${ }^{7}$ Department of Nuclear Imaging and Bio imaging, Tata Memorial Centre, Mumbai, India
\end{abstract}

Twitter Abhilash Gavarraju @AbhiG76898224

Contributors Study concept and design: VM, IM; Selection accrual and consenting of patients: VM, IM, AG, SS, RK, TT, AM; Patient examination and clinical evaluation: VM, IM, AG, SS, RK, TT, AM, GB, GP, MP; Radiological evaluation: PP, VR, AA; Pathological evaluation: SM; Statistical Analysis and Interpretation: SK; Data collection and Master File maintenance: SK. All authors have read and approved the manuscript.

Funding This ongoing study is funded by an intramural grant from Tata Memorial Centre, Mumbai (TRAC/0518/900271/001). No industry or pharmaceutical support has been received. The granting bodies are not involved in data collection or analysis.

Competing interests None declared.

Patient consent for publication Not required.

Ethics approval Signature of the informed consent will be obtained from all patients before inclusion in the study. This ongoing study was approved by Institutional Ethics Committee of Tata Memorial Hospital, Mumbai (TMC IRB Project No: -271/CTRI No: CTRI/2018/05/014054). This study was approved by Institutional Ethics Committee of Tata Medical Center, Kolkata (2018/TMC/134/IRB32). This 
trial is registered prospectively with CTRI/2018/05/014054 (http://ctri.nic.in) (REF/2018/05/019975) on 24 May 2018.

Provenance and peer review Not commissioned; externally peer reviewed.

Open access This is an open access article distributed in accordance with the Creative Commons Attribution Non Commercial (CC BY-NC 4.0) license, which permits others to distribute, remix, adapt, build upon this work non-commercially, and license their derivative works on different terms, provided the original work is properly cited, appropriate credit is given, any changes made indicated, and the use is non-commercial. See: http://creativecommons.org/licenses/by-nc/4.0/.

\section{ORCID iDs}

Vedang Murthy http://orcid.org/0000-0003-4233-6437

Abhilash Gavarraju http://orcid.org/0000-0002-1498-179X

Tejshri Telkhade http://orcid.org/0000-0002-1262-9311

\section{REFERENCES}

1 Cooperberg MR, Broering JM, Carroll PR. Time trends and local variation in primary treatment of localized prostate cancer. JCO 2010;28:1117-23.

2 Punnen S, Cooperberg MR. The epidemiology of high-risk prostate cancer. Curr Opin Urol 2013;23:331-6.

3 Jain S, Saxena S, Kumar A. Epidemiology of prostate cancer in India. Meta Gene 2014:2:596-605.

4 Hariharan K, Padmanabha V. Demography and disease characteristics of prostate cancer in India. Indian J Urol 2016;32:103.

5 Hebert JR, Ghumare SS, Gupta PC. Stage at diagnosis and relative differences in breast and prostate cancer incidence in India: comparison with the United States. Asian Pac J Cancer Prev 2006; 7:547-55

6 Vogelius IR, Bentzen SM. Meta-Analysis of the alpha/beta ratio for prostate cancer in the presence of an overall time factor: bad news, good news, or no news? Int J Radiat Oncol Biol Phys 2013;85:89-94.

7 Dearnaley D, Syndikus I, Sumo G, et al. Conventional versus hypofractionated high-dose intensity-modulated radiotherapy for prostate cancer: preliminary safety results from the $\mathrm{CHHiP}$ randomised controlled trial. Lancet Oncol 2012:13:43-54.

8 Widmark A, Gunnlaugsson A, Beckman L, et al. Ultrahypofractionated versus conventionally fractionated radiotherapy for prostate cancer: 5-year outcomes of the HYPO-RT-PC randomised, non-inferiority, phase 3 trial. The Lancet 2019;394:385-95

9 Musunuru HB, D'Alimonte L, Davidson M, et al. Phase 1-2 study of stereotactic ablative radiotherapy including regional lymph node irradiation in patients with high-risk prostate cancer (SATURN): early toxicity and quality of life. Int $J$ Radiat Oncol Biol Phys 2018;102:1438-47.
10 Murthy V, Gupta M, Mulye G, et al. Early results of extreme Hypofractionation using stereotactic body radiation therapy for highrisk, very high-risk and node-positive prostate cancer. Clin Oncol 2018;30:442-7.

11 Salembier C, Villeirs G, De Bari B, et al. ESTRO ACROP consensus guideline on CT- and MRI-based target volume delineation for primary radiation therapy of localized prostate cancer. Radiother Oncol 2018;127:49-61.

12 Murthy V, Sinha S, Kannan S, et al. Safety of prostate stereotactic body radiation therapy after transurethral resection of prostate (TURP): a propensity score matched pair analysis. Pract Radiat Oncol 2019;9:347-53

13 Bekelman JE, Deye JA, Vikram B, et al. Redesigning radiotherapy quality assurance: opportunities to develop an efficient, evidencebased system to support clinical trials--report of the National Cancer Institute Work Group on Radiotherapy Quality Assurance. Int J Radiat Oncol Biol Phys 2012;83:782-90.

14 Bauman G, Ferguson M, Lock M, et al. A phase 1/2 trial of brief androgen suppression and stereotactic radiation therapy (FASTR) for high-risk prostate cancer. Int J Radiat Oncol Biol Phys 2015;92:856-62.

15 Quon HC, Ong A, Cheung P, et al. Once-Weekly versus everyother-day stereotactic body radiotherapy in patients with prostate cancer (patriot): a phase 2 randomized trial. Radiother Oncol 2018;127:206-12.

16 Brand DH, Tree AC, Ostler P, et al. Intensity-Modulated fractionated radiotherapy versus stereotactic body radiotherapy for prostate cancer (PACE-B): acute toxicity findings from an international, randomised, open-label, phase 3, non-inferiority trial. Lancet Oncol 2019;20

17 Kang J, Katz AJ. Management of high-risk prostate cancer with 5-treatment stereotactic body radiotherapy: long-term results. JCO 2017;35:94

18 King CR, Freeman D, Kaplan I, et al. Stereotactic body radiotherapy for localized prostate cancer: pooled analysis from a multiinstitutional Consortium of prospective phase II trials. Radiother Oncol 2013;109:217-21.

19 Davis J, Sharma S, Shumway R, et al. Stereotactic body radiotherapy for clinically localized prostate cancer: toxicity and biochemical disease-free outcomes from a multi-institutional patient registry. Cureus 2015;7:e395

20 Ricco A, Manahan G, Lanciano R, et al. The comparison of stereotactic body radiation therapy and intensity-modulated radiation therapy for prostate cancer by NCCN risk groups. Front Oncol 2016;6:184.

21 Katz A, Kang J. Stereotactic body radiation therapy for localized prostate cancer: risk stratification for intermediate- and high-risk patients. Int J Radiat Oncol Biol Phys 2016;96:E250.

22 Koskela K, Palmgren J-E, Heikkilä J, et al. Hypofractionated stereotactic body radiotherapy for localized prostate cancer - first Nordic clinical experience. Acta Oncol 2017;56:978-83. 